Eur. J. Biochem. 49, 469-475 (1974)

\title{
Control of Fatty-Acid Biosynthesis by Long-Chain Acyl CoAs and by Lipid Membranes
}

\author{
Manfred SUMPER \\ Max-Planck-Institut für Biophysikalische Chemie, Göttingen-Nikolausberg
}

(Received June 27/September 3, 1974)

The possibility of a regulatory role of long-chain acyl-CoAs was studied in a system synthesizing palmitoyl-CoA and stearoyl-CoA in vitro. This system contained acetyl-CoA carboxylase (yeast), fatty acid synthetase (yeast) and lipid membranes as acceptors for the long-chain acyl-CoA compounds. In this system acetyl-CoA carboxylase is controlled by long-chain acyl-CoAs, whereas this is not true for fatty acid synthetase. The degree of inhibition of acetyl-CoA carboxylase depends strongly upon the capacity of the lipid membrane to incorporate long-chain acyl-CoAs. This capacity is not fixed but is a function of different parameters such as surface charge of the membrane. The effects of inositol deficiency in vivo (overproduction of lipids) and the shift to shorter chain lengths of the fatty acids synthesized under anaerobic conditions are discussed and interpreted.

Several authors [1 -5$]$ have implicated palmitoyl$\mathrm{CoA}$ in the control of various pathways, especially in the control of fatty acid biosynthesis, while others, notably Srere [6], Taketa and Pogell [7], Dorsey and Porter [8] have suggested the possibility of a detergent action by palmitoyl-CoA on various enzymes. For these reasons the latter authors considered palmitoyl CoA a very unlikely candidate for a physiological agent of control.

Irrespective of these difficulties, the problem exists in fatty acid biosynthesis to transport palmitoyl$\mathrm{CoA}$ molecules from their place of synthesis (fatty acid synthetase) to the subsequent enzymes of lipid biosynthesis. In a recent paper [9] it was shown that the products of fatty acid synthetase, palmitoyl-CoA and stearoyl-CoA remain attached to the multienzyme complex and inhibit its enzymatic activity unless acceptors for the long-chain acyl-CoA compounds are present. Thus a free diffusion of palmitoyl-CoA molecules within the cytoplasm is excluded. It was further shown that palmitoyl-CoA can be transferred from the fatty acid synthetase into natural membranes or into lipid bilayers. It was suggested that lateral

Enzymes. Acetyl-CoA carboxylase (EC 6.4.1.2); catalase (EC 1.11.1.6); DNase (EC 3.1.4.5); glucose-6-phosphate dehydrogenase (EC 1.1.1.49); hexokinase (EC 2.7.1.1); lactate dehydrogenase (EC 1.1.1.27); lysozyme (EC 3.2.1.17); malate dehydrogenase (EC 1.1.1.37); 3-phosphoglycerate kinase (EC 2.7.2.3); pyruvate kinase (EC 2.7.1.40). diffusion within the plane of the membrane would be the mechanism of transport of palmitoyl-CoA molecules to the subsequent enzymes of lipid synthesis, which are known to be firmly associated with membranes [10].

It is the objective of this paper to reexamine the question of a control function of long-chain acyl-CoA compounds with special attention to the following postulates.

a) Only those enzymes with an affinity for palmitoyl-CoA comparable to that of lipid membranes may be controlled by long-chain acyl-CoAs. Only such enzymes can compete with membranes for longchain acyl-CoA molecules.

b) To be physiologically meaningful, one would expect that enzymes whose activities are sensitive to palmitoyl-CoA are affected reversibly.

These questions were studied using a system synthesizing long-chain acyl-CoAs in vitro. This system contained acetyl-CoA carboxylase (yeast), fatty acid synthetase (yeast) and lipid bilayers as acceptors. It was demonstrated that acetyl-CoA carboxylase is likely to be controlled in vivo by long-chain acyl-CoAs as judged by the above criteria, whereas this is not true for fatty acid synthetase. Furthermore, evidence is presented for a close correlation between the rate of fatty acid synthesis and the lipid composition of the membrane. 


\section{MATERIALS AND METHODS}

\section{Chemicals}

Coenzyme A, NADPH and enzymes for analytical determinations were purchased from Boehringer Mannheim GmbH (Mannheim, Germany). Dimyristoyllecithin was a product from Koch-Light, dimyristoyl phosphatidic acid was a gift from Dr Eibl. The lipids were ultrasonically dispersed to yield optically clear solutions.

\section{Preparation of Substrates and Long-Chain Acyl-CoA Derivatives}

Acetyl-CoA was synthesized from acetic anhydride and $\mathrm{COA}$ [11] and its concentration determined according to Buckel and Eggerer [12]. Malonyl-CoA was prepared and assayed as described in [13]. Palmitoyl-CoA and palmitoleyl-CoA were synthesized via their mixed anhydrides [14] and purified according to the method of Schultz [15]. The concentrations of the long-chain acyl-CoA compounds were determined by their absorbance at 260 and $232 \mathrm{~nm}$ [16].

\section{Enzymes}

The purification and assay of fatty acid synthetase from yeast followed procedures described elsewhere [17]. Acetyl-CoA carboxylase from yeast was purified as described in [18]. The kinetics of fatty acid synthesis was measured by following NADPH consumption using a Cary 14. A total of 14 NADPH molecules is consumed for 1 palmitoyl-CoA.

\section{Standard Assay Mixture for Fatty-Acid Synthesis}

The standard assay mixture contained per $\mathrm{ml}$ : $100 \mu \mathrm{mol}$ potassium phosphate $\mathrm{pH} 7.5,0.2 \mu \mathrm{mol}$ NADPH, $2 \mu \mathrm{mol}$ ATP, $10 \mu \mathrm{mol} \mathrm{MgCl}_{2}, 10 \mu \mathrm{mol}$ $\mathrm{KHCO}_{3}$.

\section{RESULTS}

Fig. 1 presents the kinetics of fatty acid synthesis in our system under different conditions. Curve I: acetyl-CoA carboxylase and fatty acid synthetase together with their respective substrates (acetyl-CoA, ATP, $\mathrm{KHCO}_{3}$ and NADPH) were present, however acceptors for palmitoyl-CoA were absent. In this case the rate of synthesis decreases rapidly to zero after a short period of activity (in the following denoted as "active period"). Curve II: upon addition of lipid bilayers, a prolonged period of synthesis is observed. The amount of palmitoyl-CoA and stearoyl-CoA

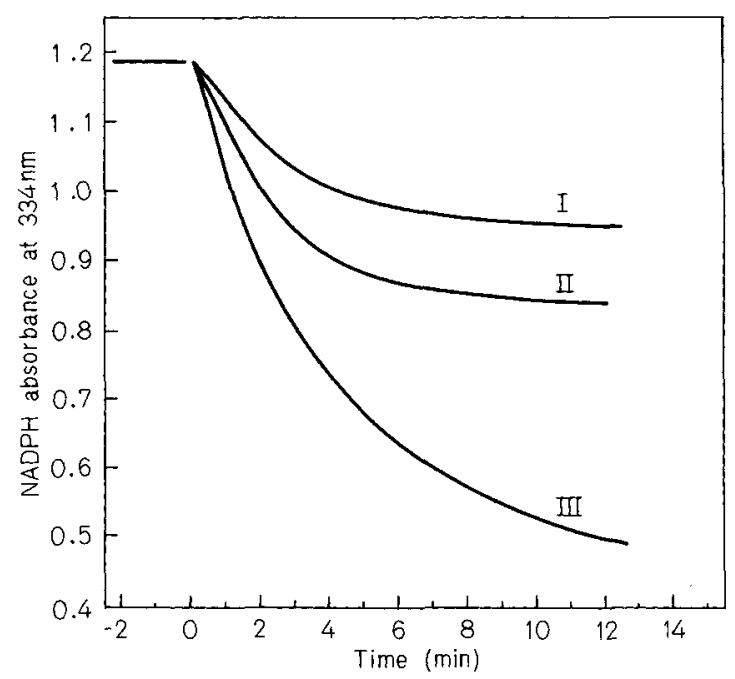

Fig. 1. Rate and extent of fatty acid synthesis in the presence or absence of acetyl-CoA carboxylase and with lipid bilayers as acceptors for the long-chain acyl-CoA products. Curve (I): the standard incubation mixture (as in Materials and Methods) contained in addition $3.4 \mu \mathrm{g}$ acetyl-CoA carboxylase $(4000 \mathrm{mU} / \mathrm{mg})$ and $30 \mu \mathrm{g}$ fatty acid synthetase $(1200 \mathrm{mU} / \mathrm{mg})$. No lipid was added. At zero time the reaction was initiated by the addition of $0.1 \mu \mathrm{mol}$ acetyl-CoA. Reaction was at $25^{\circ} \mathrm{C}$. Curve (II): identical conditions as for curve (I), but in the presence of $5 \mu \mathrm{M}$ dimyristoyllecithin. Curve(III): identical conditions as for curve (II), but acetyl-CoA carboxylase was replaced by its product malonyl-CoA $(0.1 \mu \mathrm{mol})$

synthesized during the active period increases linearly with the lipid added (not shown). Curve III : acetylCoA carboxylase was replaced by its product malonylCoA while the other conditions were identical to those of the experiment of curve II. The main difference to the experiment of curve II is the about four-fold increase of long-chain acyl-CoA formation. As is known from previous work (for review see [19]), fatty acid synthetase as well as acetyl-CoA carboxylase are inhibited by long-chain acyl-CoAs. The lower extent of synthesis in curve II suggests that acetyl-CoA carboxylase is much more sensitive to long-chain acyl-CoAs than fatty acid synthetase. Most probably acetyl-CoA carboxylase has a high affinity for palmitoyl-CoA and is already completely inhibited at a low occupation level of the membrane which does not affect the activity of fatty acid synthetase. The results presented in Fig. 2 support this interpretation. Increasing amounts of palmitoyl-CoA were added to the complete system (acetyl-CoA carboxylase, fatty acid synthetase and lipid bilayers). Curve III demonstrates that the activity of fatty acid synthetase is completely unaffected by palmitoyl-CoA within the concentration range used. Nevertheless, the rate of fatty acid synthesis decreases (curve II). Curve I shows the inhibition of the fatty acid-synthesizing 


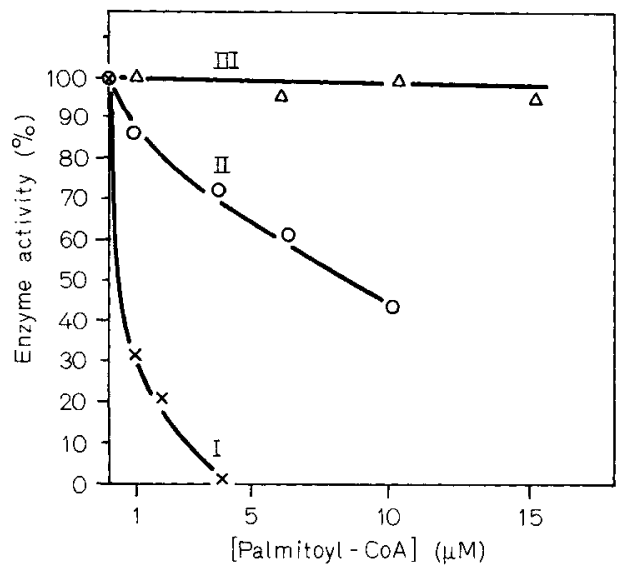

Fig. 2. The inhibitory effect of palmitoyl-CoA on the fatty acidsynthesizing system containing acetyl-CoA carboxylase and fatty acid synthetase. The standard incubation mixtures contained in addition $8.4 \mu \mathrm{g}$ acetyl-CoA carboxylase $(4000 \mathrm{mU} /$ $\mathrm{mg}), 30 \mu \mathrm{g}$ fatty acid synthetase $(1200 \mathrm{mU} / \mathrm{mg}), 0.1 \mu \mathrm{mol}$ acetyl-CoA and palmitoyl-CoA as indicated. Curve (I): in hibition in the absence of lipid. Curve (II): inhibition in the presence of $0.1 \mathrm{mM}$ dimyristoyllecithin. Curve (III): inhibitory effect on the fatty acid synthetase in the presence of $0.1 \mathrm{mM}$ dimyristoyllecithin: incubation mixture as in (II), but in addition $0.1 \mu \mathrm{mol}$ malonyl-CoA.

system in the absence of lipid bilayers. The conclusion to be drawn from these results is that acetyl-CoA carboxylase must be the site of action of palmitoylCoA.

Our results so far demonstrate that acetyl-CoA carboxylase competes with lipid bilayers for longchain acyl-CoAs. Therefore one would expect that acetyl-CoA carboxylase can also accept palmitoylCoA molecules from the fatty acid synthetase complex. The following assay was used to demonstrate this ability of acetyl-CoA carboxylase. Increasing amounts of acetyl-CoA carboxylase were added to a fatty acid synthetase solution (containing the appropriate substrates) in the presence of malonyl-CoA; this ensures that acetyl-CoA carboxylase is not an essential part of the fatty acid synthesizing system and thus allows one to assay the palmitoyl-CoA-accepting capacity of acetyl-CoA carboxylase. As is seen from Fig. 3, the extent of long-chain acyl-CoA synthesis increases linearly with the amount of acetyl-CoA carboxylase protein added.

In order to establish whether this acceptor property is specific for acetyl-CoA carboxylase a series of proteins was checked for their ability to accept palmitoyl-CoA molecules from fatty acid synthetase. The transfer was measured optically using the assay

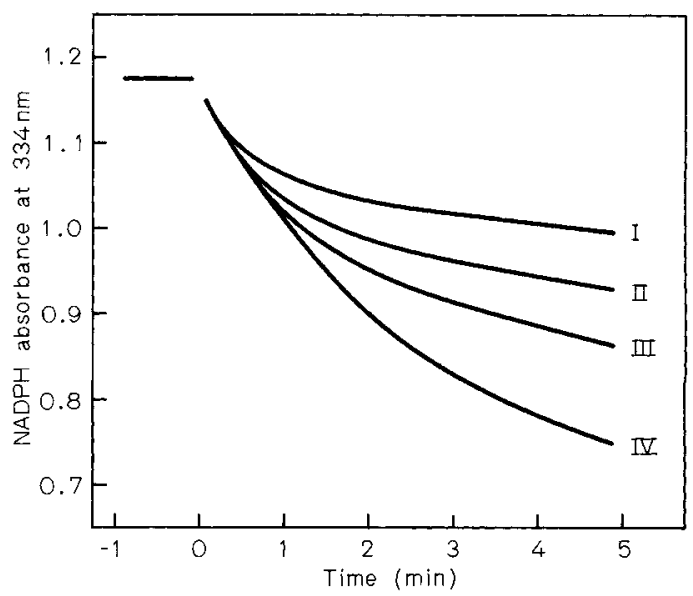

Fig. 3. Acetyl-CoA carboxylase as an acceptor for long-chain acyl-CoA products from fatty acid synthetase. The incubation mixture $(1 \mathrm{ml})$ contained: $100 \mu \mathrm{mol}$ potassium phosphate $\mathrm{pH} 7.5,0.2 \mu \mathrm{mol}$ NADPH, $0.1 \mu \mathrm{mol}$ acetyl-CoA and $20 \mu \mathrm{g}$ fatty acid synthetase. The reaction was initiated by the addition of $0.1 \mu \mathrm{mol}$ malonyl-CoA. $25^{\circ} \mathrm{C}$. Curve (I): incubation mixture without acetyl CoA carboxylase protein. Curves (II)-(IV): increasing amounts of acetyl-CoA carboxylase protein added to the incubation mixture: (II) $3.4 \mu \mathrm{g}$; (III) $6.8 \mu \mathrm{g}$; (IV) $13.6 \mu \mathrm{g}$

Table 1. Acceptor properties of various proteins for long-chain acyl-CoA products from fatty acid synthetase

Assay was performed as described in the legend to Fig. 3. Acceptor capacity is expressed in a percentage of the acetylCoA carboxylase acceptor capacity (compared on a weight basis)

\begin{tabular}{lc}
\hline Protein & Acceptor capacity \\
\hline & $\%$ \\
3-Phosphoglycerate kinase & 0 \\
Lysozyme & 0 \\
Lactate dehydrogenase & 0 \\
DNase & 6 \\
Malate dehydrogenase & 0 \\
Hexokinase & 13 \\
Glucose-6-phosphate dehydrogenase & 27 \\
Catalase & 0 \\
Pyruvate kinase & 0 \\
Cytochrome $c$ & 5 \\
Serum albumin & 55 \\
Acetyl-CoA carboxylase & 100 \\
\hline
\end{tabular}

described above (Fig. 3). The results are summarized in Table 1. None of eleven proteins chosen at random showed an affinity for palmitoyl-CoA similar to that of acetyl-CoA carboxylase. Even serum albumin, a carrier of fatty acids in serum, is only half as effective 


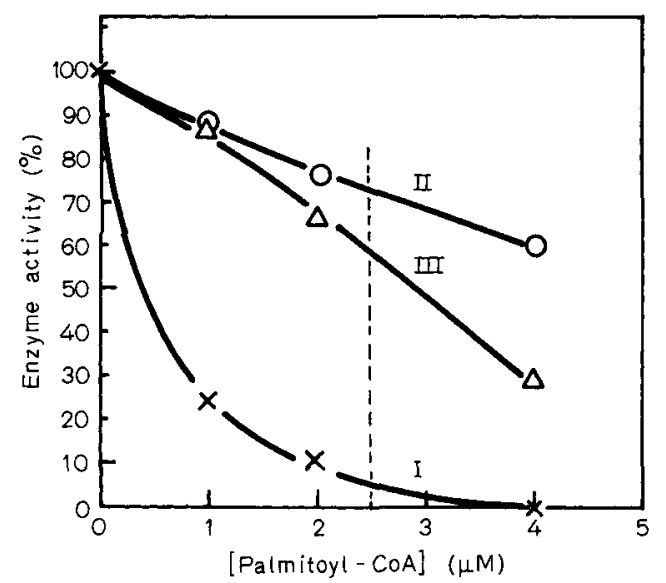

Fig. 4. Reversibility of the inhibitory effect of palmitoyl-CoA on the fatty acid-synthesizing system. The standard incubation mixture contained in addition $8.4 \mu \mathrm{g}$ acetyl-CoA carboxylase $(4000 \mathrm{mU} / \mathrm{mg}), 30 \mu \mathrm{g}$ fatty acid synthetase $(1200 \mathrm{mU} / \mathrm{mg})$, $0.1 \mu \mathrm{mol}$ acetyl-CoA and palmitoyl-CoA as indicated. Curve (I) : inhibition in the absence of lipid. Curve (II): inhibition in the presence of $0.2 \mathrm{mM}$ dimyristoyllecithin. Curve (III): conditions as for curve (I), but after the inhibition of the system, $0.2 \mathrm{mM}$ dimyristoyllecithin was added to the mixture and after incubation for $2 \mathrm{~min}$ the restoration of fatty acidsynthesizing activity was measured

as acetyl-CoA carboxylase (judged on a weight basis). Compared on a molar basis (capacity per protein molecule), serum albumin is only $5 \%$ as effective as acetyl-CoA carboxylase. Six proteins had absolutely no affinity for long-chain acyl-CoA molecules, while two showed small acceptor properties. Interestingly, these two enzymes are key enzymes in sugar metabolism and one of them (glucose-6-phosphate dehydrogenase) is believed to be regulated by palmitoyl-CoA [20]. It was not checked, however, whether these two enzymes may also compete with lipid membranes for palmitoyl-CoA as is the case with acetyl-CoA carboxylase.

Attachment of long-chain acyl-CoA compounds to acetyl-CoA carboxylase impairs the enzymatic activity. The following experiment was designed to check the reversibility of this inhibition. Acetyl-CoA carboxylase was inhibited by adding sequential amounts of palmitoyl-CoA (in the absence of lipid bilayers). After a period of $3 \mathrm{~min}$ lipid bilayers were added and the enzymatic activity was determined $2 \mathrm{~min}$ later. The references used were analogous experiments where palmitoyl-CoA was added in the presence (curve II) and absence of lipid bilayers (curve I). As demonstrated by Fig. 4, the inhibition of acetyl-CoA carboxylase was reversible within the

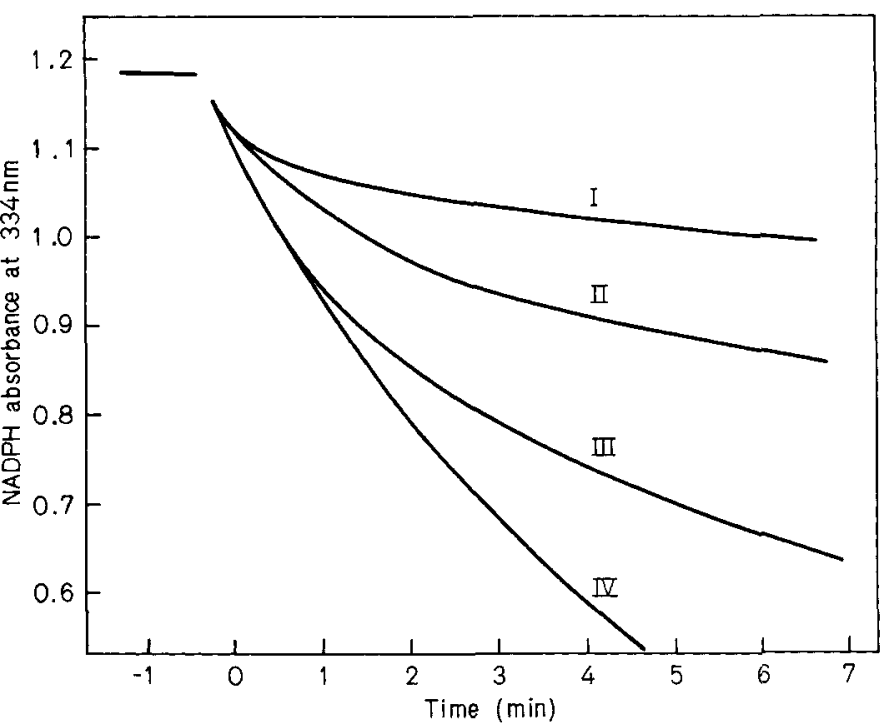

Fig. 5. The acceptor capacity of dimyristoyl phosphatidic acid at different ionic conditions. The incubation mixture $(1 \mathrm{ml})$ contained: 40 or $100 \mu \mathrm{mol}$ potassium phosphate $\mathrm{pH} 7.5$, $0.2 \mu \mathrm{mol} \mathrm{NADPH}, 0.1 \mu \mathrm{mol}$ acetyl $\mathrm{CoA}$ and $20 \mu \mathrm{g}$ fatty acid synthetase $(1200 \mathrm{mU} / \mathrm{mg})$. The reaction was initiated by the addition of $0.1 \mu \mathrm{mol}$ malonyl-CoA. Reaction at $25^{\circ} \mathrm{C}$. Curve (I): no lipid added. Curve (II): $40 \mathrm{mM}$ potassium phosphate and $0.05 \mathrm{mM}$ dimyristoylphosphatidic acid. Curve (III) : $100 \mathrm{mM}$ potassium phosphate and $0.05 \mathrm{mM}$ dimyristoylphosphatidic acid. Curve (IV): conditions as for (II), but in addition $10 \mathrm{mM} \mathrm{Mg}^{2+}$

applied palmitoyl-CoA concentration range. In vivo, the upper limit of the palmitoyl-CoA concentration is determined by the complete inhibition of acetylCoA carboxylase. Under the conditions of our experiment this situation was reached (in the absence of lipids) at about 2.5 to $3 \mu \mathrm{M}$ palmitoyl-CoA (dotted line in Fig. 4).

\section{Effect of Negative Charges on the Acceptor Properties of Lipid Bilayers}

At physiological $\mathrm{pH}$ the palmitoyl-CoA molecule carries 3 to 4 negative charges. Therefore one would expect that the incorporation of long-chain acyl-CoAs into a membrane is governed by the surface charge and by the ionic strength. Fig. 5 shows the rate and extent of fatty acid synthesis in the presence of dimyristoylphosphatidic acid bilayers (at $\mathrm{pH} 7.5$ phosphatidic acid carriers 1 to 2 negative charges per molecule) for different ionic strengths (curve II and III). As expected at low ionic strength (curve II) palmitoyl$\mathrm{CoA}$ is practically not incorporated into phosphatidic acid bilayers. Only about 2 palmitoyl-CoA molecules are incorporated per 100 lipid molecules. An increased incorporation is observed at a higher ionic strength (curve III). Divalent cations like $\mathrm{Mg}^{2+}$ are even more 


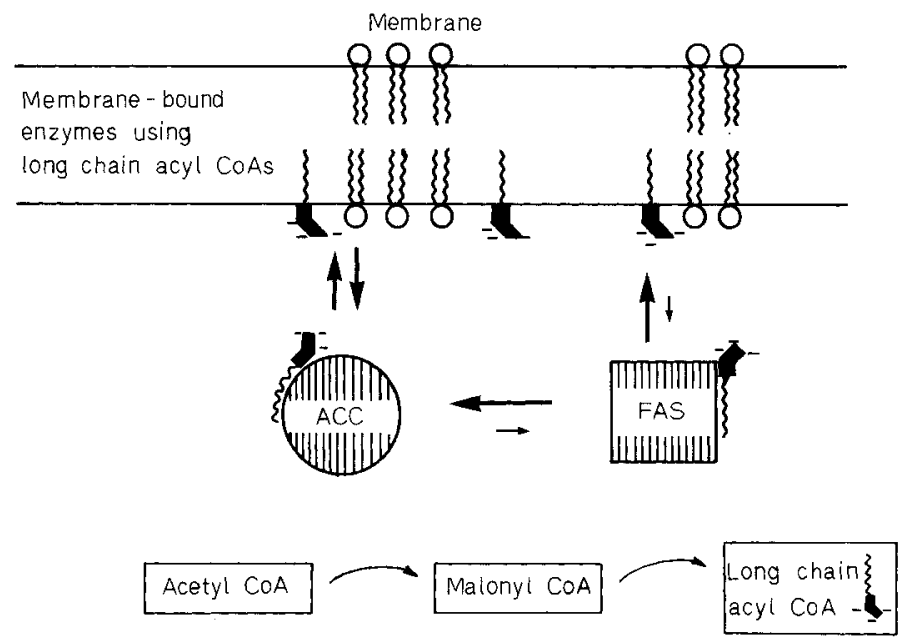

Fig. 6. Qualitative scheme for the regulation of fatty acid biosynthesis

effective. The addition of $10 \mathrm{mM} \mathrm{Mg}^{2+}$ allows a five-fold higher incorporation of palmitoyl-CoA into phosphatidic acid bilayers (curve IV). This effect is in accord with the known complex formation between phosphatidic acid bilayers and divalent cations leading to surface charge neutralization [21]. (High concentrations of $\mathrm{Mg}^{2+}$ ions also diminish the palmitoylCoA inhibition of fatty acid synthetase [F. Lynen, personal communication], possibly by favouring the formation of palmitoyl-CoA micelles.)

\section{Inhibition of Fatty-Acid Biosynthesis by Unsaturated Long-Chain Acyl-CoAs}

Fig. 7A shows the inhibition of the fatty-acidsynthesizing system by palmitoyl-CoA (curve II) and palmitoleyl CoA (curve I). This result will be used in the Discussion to explain the different chain-length patterns of fatty acids in yeast grown under aerobic or anaerobic conditions.

\section{DISCUSSION}

Fig. 6 presents a qualitative scheme for the shortterm regulation of fatty acid synthesis in yeast. The tetrameric acetyl-CoA carboxylase supplies the multienzyme complex fatty acid synthetase with malonylCoA. The long-chain acyl-CoA products of fatty acid synthetase are transferred into the membrane directly or via acetyl-CoA carboxylase ("carrier" action). Lateral diffusion within the plane of the membrane would be the mechanism by which the long-chain acyl-CoA molecules are transported to the subsequent membrane-bound enzymes of lipid biosynthesis. This mechanism avoids all complications due to unspecific associations of palmitoyl-CoA with soluble proteins of the cytoplasm (detergent properties of palmitoyl-CoA). Acetyl-CoA carboxylase competes with the lipid membrane for the longchain acyl CoAs. (The transfer of palmitoyl-CoA between membrane and acetyl-CoA carboxylase is reversible.) Thus the inhibition of acetyl-CoA carboxylase by palmitoyl-CoA is directly correlated to the level of occupation of the membrane. Interestingly, a level of palmitoyl-CoA causing $80 \%$ inhibition of acetyl-CoA carboxylase does not affect the enzymatic activity of fatty acid synthetase. Thus the inhibition of fatty acid synthetase by palmitoyl-CoA in vitro [5] cannot have any relevance to the situation in vivo (see also [8]).

Two well known effects in fatty acid synthesis regulation may be interpreted using the scheme in Fig. 6. Firstly, in yeast auxotrophic for inositol, inositol deficiency leads to a decreased synthesis of phosphatidyl inositol and in line with this to an excessive synthesis of lipids, especially triacylglycerols [22]. As shown above the distribution of long-chain acylCoA molecules between membrane and acetyl-CoA carboxylase is governed by surface charges. The lower the negative surface charge the larger the capacity of the membrane for incorporation of long-chain acyl CoAs. Of the three main membrane lipids in yeast, phosphatidyl inositol (about 30\% [23]), kephaline and lecithin, only phosphatidyl inositol carries a negative charge. This would imply that inositol deficiency leads to a lower negative surface charge of the membrane. Consequently, higher occupation levels of the membrane by palmitoyl-CoA are tolerable before palmitoyl-CoA attaches to acetyl-CoA carboxylase and inhibits its activity. Thus greater amounts 


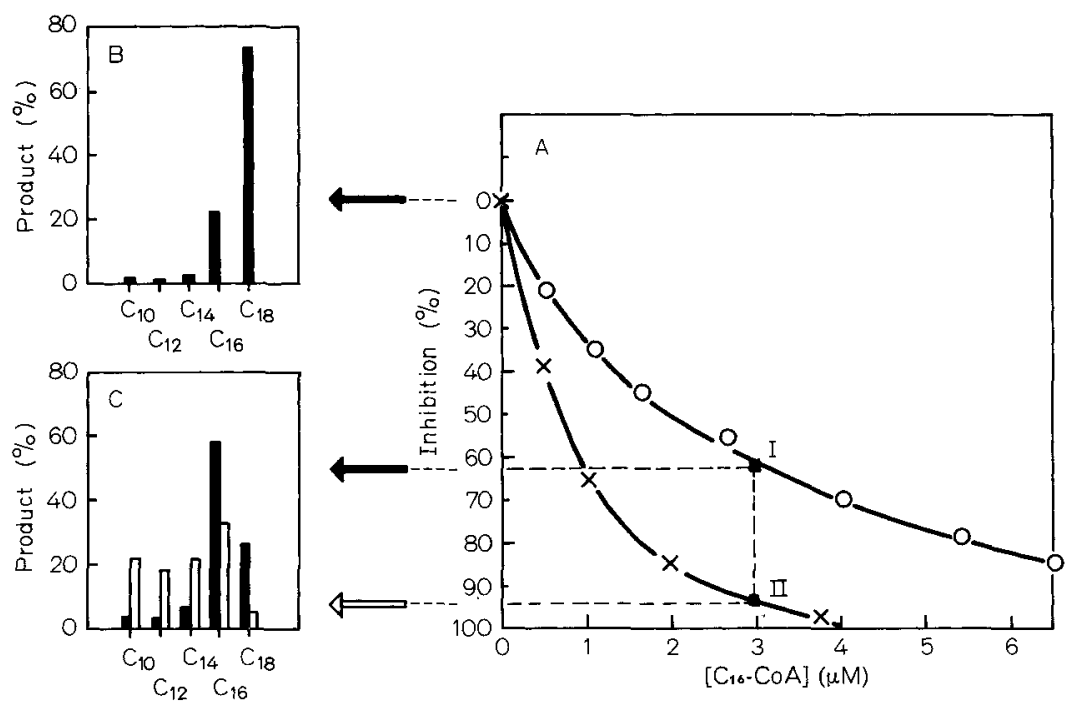

Fig. 7. Inhibitory effect of saturated and unsaturated $C_{16}-$ CoA compounds on the fatty acid-synthesizing system and the product pattern calculated for different rates of fatty acid synthesis [28]. The standard incubation mixture contained in addition $8.4 \mu \mathrm{g}$ acetyl-CoA carboxylase $(4000 \mathrm{mU} / \mathrm{mg}), 30 \mu \mathrm{g}$ fatty acid synthetase $(1200 \mathrm{mU} / \mathrm{mg}), 0.1 \mu \mathrm{mol}$ acetyl-CoA and long-chain acyl-CoA compounds as indicated. (A) Curve
(I): inhibition of fatty acid synthesis by palmitoleyl CoA; curve (II) : inhibition of fatty acid synthesis by palmitoyl CoA. (B) Calculated fatty acid chain-length pattern produced by fatty acid synthetase from yeast working at maximum rate. (C) Calculated fatty acid chain-length pattern produced by fatty acid synthetase working at $35 \%$ maximum rate (black bars) and at $6 \%$ maximum rate (white bars), respectively of fatty acid derivatives (triacylglycerols) can be produced under conditions of inositol deficiency.

A second well known effect in fatty acid synthesis regulation is the shift in the chain-length pattern of the fatty acids as a function of oxygen tension [24]. Cells grown anaerobically on media containing only traces of unsaturated fatty acids synthesize predominantly saturated fatty acids, whereby the short-chain components, decanoic $\left(\mathrm{C}_{10}\right)$ and dodecanoic $\left(\mathrm{C}_{12}\right)$ acid, make up $50 \%$ of the total fatty acids. In contrast, cells grown aerobically produce predominantly the long-chain fatty acids, palmitic $\left(\mathrm{C}_{16}\right)$ and stearic $\left(\mathrm{C}_{18}\right)$ acid.

Let us assume, that the concentrations of acetylCoA carboxylase and fatty acid synthetase within the cell are correlated in such a manner that enough acetyl-CoA carboxylase is present to allow maximum rate of fatty acid synthesis (as long as no inhibition by long-chain acyl-CoAs takes place) [25-27]. As established from studies in vitro [28], in this case a chain length pattern is obtained as shown in Fig. 7 B. The chain length profile which is actually observed, when cells are grown aerobically ( $c f$. black bars in Fig. 7C) is obtained when fatty acid synthetase operates at a reduced rate of $35-40 \%$ of its maximal activity. The quite different chain-length profile found for anaerobic growth conditions (white bars in Fig. 7C) is produced when fatty acid synthetase operates at about $10 \%$ of its maximum rate [28]. If we assume that inhibition of acetyl CoA carboxylase by longchain acyl-CoAs is the regulating step of fatty acid synthesis these different patterns can be readily explained from Fig. 7A, which shows the inhibition of acetyl-CoA carboxylase by palmitoyl-CoA (curve II) and by palmitoleyl CoA (curve I). The saturated compound is a more effective inhibitor than the unsaturated compound. Under anaerobic conditions the conversion of saturated to unsaturated acyl-CoAs is blocked and thus curve II is valid for anaerobic growth. A long-chain acyl-CoA level allowing 35$40 \%$ fatty acid synthesis in the aerobic case (curve I) allows only $5-10 \%$ synthesis in the anaerobic case (vertical dotted line in Fig. 7A) which explains exactly the different chain-length patterns observed in aerobic and anaerobic yeast.

Our system of fatty acid synthesis in vitro contained synthetic lipid bilayers instead of natural membranes. The relevance of our results to the situation in vivo is supported in investigations carried out by Bortz and Lynen [3] and more recently by Guynn et al. [29] on the short-term control of fatty acid synthesis in liver. These authors have demonstrated that palmitoyl-CoA is a physiological agent of control and also that no correlation exists between the rate of fatty acid synthesis and the concentration of a number of metabolites such as ATP, ADP, citrate, glucose, glucose 6phosphate and glycerol 3-phosphate. Only the concentrations of malonyl-CoA and long-chain acyl-CoAs 
were correlated indicating that the control of fatty acid synthesis in vivo results from inhibition of acetylCoA carboxylase by long-chain acyl-CoAs.

I thank Dr H. Träuble for helpful discussions and support. The interest of Prof. M. Eigen and Prof. F. Lynen in this work is gratefully acknowledged.

\section{REFERENCES}

1. Numa, S., Ringelmann, E. \& Lynen, F. (1965) Biochem. Z. 343, 243-257.

2. Bortz, W. M. \& Lynen, F. (1963) Biochem. Z. 337, 505509.

3. Bortz, W. M.\& Lynen, F. (1963) Biochem. Z. 339, 77-82.

4. Korchak, H. M. \& Masoro, E. J. (1964) Biochim. Biophys. Acta, 84, 750-753.

5. Lust, G. \& Lynen, F. (1968) Eur. J. Biochem. 7, 68-72.

6. Srere, P. A. (1965) Biochim. Biophys. Acta, 106, 445- 455.

7. Taketa, K. \& Pogell, B. M. (1966) J. Biol. Chem. 241, $720-726$

8. Dorsey, J. A. \& Porter, J. W. (1968) J. Biol. Chem. 243, $3512-3516$

9. Sumper, M. \& Träuble, H. (1973) FEBS Lett. 30, 29-34.

10. Gatt, S. \& Barenholz, Y. (1973) Annu. Rev. Biochem. 42, $61-85$.

11. Simon, E. J. \& Shemin, D. (1953) J. Am. Chem. Soc. 75, 2520.

12. Buckel, W. \& Eggerer, H. (1965) Biochem. Z. 343, $29-43$.
13. Eggerer, H. \& Lynen, F. (1962) Biochem. Z. 335, 540547.

14. Wieland, T.\& Bokelmann, E. (1951) Naturwissenschaften, $38,384$.

15. Schultz, J. (1968) Doctoral Dissertation, University of Munich.

16. Seubert, W. (1960) Biochem. Prep. 8, 80.

17. Lynen, F. (1969) Methods Enzymol. 14, 17-33.

18. Sumper, M. \& Riepertinger, C. (1972) Eur. J. Biochem. $29,237-248$.

19. Volpe, J. J. \& Vagelos, P. R. (1973) Annu. Rev. Biochem. $42,21-60$

20. Eger-Neufeldt, I., Teinzer, A., Weiss, L. \& Wieland, O. (1965) Biochim. Biophys. Res. Commun. 20, 43-48.

21. Träuble, H. \& Eibl, H. (1974) Proc. Natl Acad. Sci. U.S.A. 71, 214-219.

22. Shafei, T. \& Lewin, L. M. (1968) Biochim. Biophys. Acta, $152,787-790$.

23. Letters, R. (1968) Bull. Soc. Chim. Biol. 50, 1385- 1393.

24. Jollow, D., Kellerman, G. M. \& Linnane, A. W. (1968) J. Cell Biol. 37, 221-230.

25. Chang, H. C., Seidman, J., Teebor, G. \& Lane, M. D. (1967) Biochem. Biophys. Res. Commun. 28, 682-686.

26. Majerus, P. W., Jacobs, R., Smith, M. B. \& Morris, H.P. (1968) J. Biol. Chem. 243, 3588-3595.

27. Numa, S., Nakanishi, S., Hashimoto, T., Iritani, N. \& Okazaki, T. (1970) Vitam. Horm. 28, 213-243.

28. Sumper, M., Oesterhelt, D., Riepertinger, C. \& Lynen, F. (1969) Eur. J. Biochem. 10, 377-387.

29. Guynn, R. W., Veloso, D. \& Veech, R. L. (1972) J. Biol. Chem. 247, 7325-7331.

M. Sumper, Max-Planck-Institut für Biophysikalische Chemie, D-3400 Göttingen-Nikolausberg, Am Faßberg, Federal Republic of Germany 